\title{
A pseudomolecule assembly of the Rocky Mountain elk genome reveals putative immune system gene loss near chromosomal fissions
}

Rick E Masonbrink ${ }^{1 *}$, David Alt, ${ }^{2}$ Darrel O. Bayles, ${ }^{2}$ Paola Boggiatto, ${ }^{2}$ William Edwards ${ }^{3}$, Fred Tatum,${ }^{4}$ Jeffrey Williams, ${ }^{2}$ Jenny Wilson-Welder, ${ }^{2}$ Aleksey Zimin ${ }^{5}$, Andrew Severin ${ }^{1}$, Steven Olsen ${ }^{2}$

${ }^{1}$ Genome Informatics Facility, Department of Biotech, lowa State University, Ames, IA, United States of America

${ }^{2}$ Infectious Bacterial Diseases Research Unit, National Animal Disease Center, Agricultural Research Service, U.S.

${ }^{4}$ Respiratory Diseases Research Unit, National Animal Disease Center, Agricultural Research Service, U.S.

${ }^{5}$ Department of Biomedical Engineering, Johns Hopkins University, Baltimore, MD, United States of America

* Corresponding author

28 Email: remkv6@iastate.edu 


\section{Abstract}

32 Rocky Mountain elk (Cervus canadensis) is a major reservoir for Brucella abortus in the Greater Yellowstone area,

33 which has significant economic implications to the cattle industry. Vaccination attempts against intracellular

34 bacterial diseases in elk populations have not been successful due to a negligible adaptive cellular immune

35 response. A lack of genomic resources has impeded attempts to better understand why vaccination does not

36 induce protective immunity. To overcome this limitation, PacBio, Illumina, and HiC sequencing with a total of 686-

37 fold coverage was used to assemble the elk genome into 35 pseudomolecules. A robust gene annotation was

38 generated resulting in 18,013 gene models and 33,422 mRNAs. The accuracy of the assembly was assessed using

39 synteny to the red deer and cattle genomes identifying several chromosomal rearrangements, fusions and fissions.

40 Because this genome assembly and annotation provide a foundation for genome-enabled exploration of Cervus

41 species, we demonstrate its utility by exploring the conservation of immune system-related genes. We conclude by

42 comparing cattle immune system-related genes to the elk genome, revealing nine putative gene losses in elk.

43

44

\section{Author Summary}

Brucellosis, also known as contagious abortion, is a bacterial disease that commonly affects livestock and remains prevalent in Rocky Mountain elk (Cervus canadensis). Since the 1920's the USDA has led a program to eradicate Brucellosis from cattle, yet wild Rocky Mountain elk continue to be a source of transmission. Attempts to vaccinate wild elk herds have been unsuccessful, due to a poor and short-lived immune response. To investigate the genetic basis for this inherent difference, we created the first genome and annotation for the Rocky Mountain elk. This genome assembly is of the highest quality and contains single linear sequences for all 35 chromosomes. In order to generate gene models, an array of RNA-Seq data and proteins from many different organ tissues and cells were used in gene prediction software. Specifically, we compare cattle immune system genes with the Rocky Mountain elk, revealing the putative loss of nine immune-system related genes in elk. 


\section{Introduction}

55 Rocky Mountain elk (Cervus canadensis) were once distributed across much of North America but now inhabit

56 remote areas. Rocky Mountain elk were nearly exterminated from the Rocky Mountains of Alberta and British

57 Columbia in the early 1900s,(1) but were restocked between 1916-1920 with elk from the Greater Yellowstone

58 Area (2-5). By 1940 elk populations expanded so greatly, that periodic culling was necessary $(3,6)$. While elk have

59 been reintroduced to many areas, the densest populations are maintained in mountainous remote areas, like the

60 Greater Yellowstone Area.

61 Elk typically avoid the presence of domesticated livestock, yet they will utilize the same grounds for grazing when

62 livestock are absent (7). This can be problematic for ranchers occupying areas near elk populations like the Greater

63 Yellowstone Area. Elk are known reservoirs for brucellosis, (Brucella abortus) a disease that is highly contagious

64 and poses a risk to livestock and humans (8-10). Because of the potential for causing abortion in cattle, the USDA

65 used vaccines and serologic testing to nearly eradicate $B$. abortus from domestic herds (11). Yet in the last 15

66 years, over 20 cases of transmission to cattle have been traced to wild elk populations in the Greater Yellowstone

67 Area. Attempts to establish long-term immunity through vaccination have proven unfruitful, as elk have negligible

68 adaptive cellular immune responses to existing Brucella vaccines (12). Because the eradication of $B$. abortus from

69 cattle herds can cost hundreds of thousands of dollars and current tools make it unfeasible to control infection in

70 wild elk, there is a need to dissect the genetic nature of limited immune responses in elk. With advances in

71 sequencing technology (PacBio, Illumina and $\mathrm{HiC}$ ), we are now able to investigate difference in adaptive immune

72 response at the genomic level by examining the presence and absence of immune system-related genes. Here, we

73 report a chromosomal level reference genome assembly and annotation of the Rocky Mountain elk and perform a

74 preliminary investigation of immune gene loss between elk and cattle. Our results suggest a mechanism for gene

75 loss of immune related genes through major chromosomal rearrangement and fusion. 


\section{Methods}

77

78

79

80

81

82

83

84

85

86

87

88

89

90

91

92

93

94

95

96

97

98

99

100

\section{Animal Selection}

A male Rocky Mountain elk from a long term captive herd in Minnesota was selected for sequencing. The research protocol was approved by the National Animal Disease Center Animal Care and Use committee and all animals under the protocol were maintained in accordance with animal care regulations.

\section{Sequencing}

For the initial contig assembly we generated a hybrid data set with Illumina PCR-free 150bp paired end reads and PacBio RSII reads produced with P6-C4 chemistry. Chicago and HiC libraries were prepared as described previously $(13,14)$. Both Chicago and HiC libraries were prepared similarly, though HiC libraries were nuclear-fixed. Briefly, formaldehyde-fixed chromatin was digested with Dpnll, and 5' overhangs were sealed with biotinylated nucleotides. Blunt ends were ligated, followed by crosslink were reversed for DNA purification from protein. We then removed biotin that was not internal to ligated fragments. DNA was sheared to a mean length of $\sim 350 \mathrm{bp}$ for library construction with NEBNext Ultra enzymes and Illumina-compatible adapters. Biotin-containing fragments were isolated using streptavidin beads before PCR enrichment of the libraries. Both Chicago and HiC libraries were sequenced on an Illumina HiSeqX at 2x150bp, attaining totals of 470 million and 500 million reads, respectively.

\section{Genome Assembly}

An initial genome assembly was generated with Masurca version 3.2.3 (15), attaining a 2,559.8 Mbp genome size in 29,125 contigs with N50 size of 1,224,689bp. Dovetail Genomics scaffolded this assembly using an iterative HiRise analysis informed via alignments of Chicago and then HiC libraries with a modified SNAP aligner (http://snap.cs.berkeley.edu). This assembly contained 2,560.5 Mb, with an L90 of 31 scaffolds, and a N90 of 43.374 Mb. 1,004,453,472 Chicago and HI-C reads were used to scaffold this Dovetail assembly with a Juicer 1.5.6, 3D-DNA 180922, and JuiceBox 1.9.8 (16, 17). Reads were extracted from bam files with Picard 2.9.2 (18). The Dovetail assembly was masked using RepeatModeler 4.0.7 (19) and RepeatMasker 1.0.8 (20), prior to the alignment of $\mathrm{HI}-\mathrm{C}$ reads with BWA mem 0.7.17(21). Alignments were processed using Juicer, 3D-DNA(22), and Juicebox $(16,17)$. The Juicebox assembly strategy consisted of: manually placing all contigs greater than $10 \mathrm{~kb}$, 
101 incorporating scaffolds at the highest HI-C signal, placing scaffolds in non-repetitive regions when HI-C signal was

102 equal between a repetitive and non-repetitive region, repeats were clustered whenever possible, and only obvious

103 mis-joins were edited. The initial Juicebox scaffolding created 34 pseudomolecules, which was then compared to

104 the Cervus elaphus hippelaphus genome assembly (GCA_002197005.1)(23)to reveal the merger of the $X$ and $Y$

105 chromosomes. A BLASTn (24) of the C. elaphus hippelaphus genome sequence was used to identify coordinates,

106 allowing the correct separation the $X$ and $Y$ chromosome via the heatmap in Juicebox. The 3D-DNA assembly

107 finished with 22,557 scaffolds.

108 The contigs that could not be integrated into the pseudomolecules were eliminated based on repetitiveness, 109 duplicated heterozygous contigs, RNA-seq mapping potential, and contig size (>500 bp). BEDTools 2.25 .0 (25) was 110 used to merge coordinates from mapping these contigs to the pseudomolecules with BLAST+2.9 (score $>300$ ) and

111 RepeatMasker 1.0.8 (20) masking coordinates. 22,065 contigs were eliminated that were less than $1 \mathrm{~kb}$, had at least $11290 \%$ query coverage, and lacked a single unique mapping RNA-seq read, leaving 35 pseudomolecules, 457 contigs, 113 and a mitochondrial genome.

114 The assembly was polished with Pilon 1.23 (26) using CCS PacBio reads and paired end Illumina DNA-seq. CCS

115 PacBio reads were created from the PacBio subreads using bax2bam (27)and Bamtools 2.5.1 (28) and then aligned 116 using Minimap 2.6 (29). Paired end reads were aligned using Hisat2 2.0.5 (30), followed by bam conversion and 117 sorting with Samtools 1.9 (31). Due to uneven and excessive coverage in repetitive regions, paired end alignments 118 were set at a max coverage of 30x using jvarkit (32). Due to the excessive repetitiveness of Chromosome_14, $11950 \mathrm{Mbp}$ of this chromosome was not polished.

120 After polishing, another round of small contig elimination was performed by merging RepeatMasker (20)

121 coordinates and coordinates from BLAST+ 2.9 (24) (score $>300$, width $1000 \mathrm{bp}$ ) to the pseudomolecules with

122 Bedtools 2.25.0 (25). If $90 \%$ of query length was repetitive and contained within the pseudomolecules, it was

123 eliminated. BlobTools 1.11 (33) was run with PacBio subread alignments to the genome, and contigs annotated

124 with BLAST (24) to the NT database(Supplemental Figure 1). All scaffolds passed contamination screening, resulting 125 in a final assembly containing 35 pseudomolecules, 151 contigs, and the mitochondria. 


\section{Mitochondrial identification and annotation}

127 BLAST+ 2.9 (24) was used to identify the mitochondrial genome by querying the mitochondrial scaffold of the $C$. elaphus hippelaphus GCA_002197005.1(23). Though the mitochondrial genome was identified, it contained three juxtaposed mitochondrial genome duplications. The scaffold was manually corrected with Samtools 1.9 (31). Genes were annotated in the mitochondrial genome using the Mitos2 webserver (34) with RefSeq 89 Metazoa, a genetic code of 2, and default settings.

\section{Repeat prediction}

133 A final version of predicted repeats was obtained using EDTA 1.7.9 (35) and RepeatModeler 1.0.8 (19) with

134 RepeatMasker 4.1.0(20).

A total of 753,228,475 RNA-seq reads aligned to the genome using Hisat2 2.0.5 (30) followed by bam conversion

137 and sorting with Samtools 1.9 (31). RNA-seq read counts were obtained using Subread 1.5.2 (36). The alignments

138 were assembled into genome-guided transcriptomes using Trinity 2.8.4 (37-39), Strawberry 1.1.1 (40), Stringtie

139 1.3.3b $(41,42)$, and Class2 2.1.7(43). The RNA-seq alignments were also used for a gene prediction via Braker2

$140 \quad$ 2.1.4 (44)with Augustus 3.3.3 (45) on a genome soft-masked by RepeatMasker 1.0.8 (20)with a RepeatModeler

1414.0 .7 (19) library. High confidence exon splicing junctions were identified using Portcullis 1.1.2 (46). Each of these

142 assemblies were then supplied to Mikado 2.0rc6 (47) to pick consensus transcripts, while utilizing Cervus-specific

143 proteins from Uniprot (48) (downloaded 12-28-19). This mikado prediction was filtered for transposable elements

144 using Bedtools 2.25.0 intersect (25) and filtered for pseudogenes via removing genes with five or fewer mapping

145 RNA-seq reads. Using Bedtools 2.25.0 (25) intersect these filtered Mikado gene models were used to find

146 corresponding Braker2 2.1.4 (44) gene models. Both of these predictions, together with a Genomethreader 1.7.1

147 (49) alignment of Uniprot proteins from the Pecora infraorder (downloaded 02-07-20) were used for a final round

148 of Mikado gene prediction. The predicted transcripts and proteins were generated using Cufflinks (50) gffread

149 (2.2.1), and subjected to functional annotation to: Interproscan 5.27-66.0 $(51,52)$ and BLAST $(24)$ searches to NCBI 
153 Universal single copy orthologs were assessed using BUSCO $4.0(53,54)$, with the eukaryota_odb10 and cetartiodactyla_odb10 datasets in both genome and protein mode.

157 (23) and C. canadensis genome assemblies, we inferred gene orthology using BLASTp (24) at cutoffs of an e-value

158 of 1 e-5, 50\% query cover, and 70\% identity. Gene-based synteny was predicted using iAdHoRe 3.0.01 [81] with

159 prob_cutoff $=0.001$, level 2 multiplicons only, gap_size $=5$, cluster_gap $=15$, q_value $=0.01$, and a minimum of 3

160 anchor points. Synteny figures were produced using Circos (0.69.2) [82]. Dot plots were produced using MCScanX

$16120170403(56)$.

163 Immune system-related genes from Bos taurus were found in the GENE-DB database of the International

164 ImMunoGeneTics website (www.imgt.org) (57). This database is comprised of immunoglobulins (IG), T cell

165 receptors (TR) and major histocompatibility $(\mathrm{MH})$ genes from vertebrate species. A tblastn (2.9.0+) was performed against the elk and cattle genome assembiles, with an e-value cutoff of $1 \mathrm{e}-3$.

\section{Results and Discussion}


173 An initial assembly was created with MaSuRCA $(15,58)$ generating 23,302 contigs, an $L 90$ of 2,500 contigs, and an

174 N90 of 197,963bp. Through collaboration with Dovetail Genomics and then additional implementation of the

175 Juicer/JuiceBox/3D-DNA pipeline(16, 17, 22), we generated an assembly of 33 autosomes, an $X$ chromosome, a $Y$

176 chromosome, a mitochondrial genome, and 151 unincorporated contigs. We utilized synteny to identify

177 homologous chromosomes between elk and red deer, and found that nearly always, elk chromosome sizes fell

178 within the estimated size of the red deer's assembled chromosomes (23) (Supplemental Table 1). The only

179 exception is the $\mathrm{Y}$ chromosome, which was nearly twice (7.6 $\mathrm{Mb})$ the largest predicted size $(4 \mathrm{Mb})$ of the red deer

180 chromosome. We investigated all putative contaminant contigs from Blobtools (33), and ruled out contamination

181 (Supplemental Figure 1), but also took additional steps to ensure the completeness of the genome by mapping

182 reads back to the assembly. We found that we captured the majority of genome, with $90.7 \%$ and $87.3 \%$ of PacBio

183 CCS reads Illumina DNA-seq aligning to the genome (Supplemental Table 2). To evaluate the completeness of the

184 genome we ran BUSCO 4.0.2 (54) (Benchmarking Universal Single Copy Orthologs) on genome. Of the possible 255

185 and 13335 genes in the eukaryota and certartiodactyla odb10 datasets, $62 \%$ and $88.1 \%$ were complete, $2.4 \%$ and

$1862.1 \%$ were duplicated, and $3.1 \%$ and $2.1 \%$ were fragmented, and $32.5 \%$ and $9.8 \%$ were missing, respectively.

\section{Genome Annotation}

To obtain a high-quality elk gene prediction, we pursued an extensive annotation of repeats in the genome using

two repeat predictors. While EDTA (35) utilizes a comprehensive set of repeat prediction programs to create a

191 repeat annotation, Repeatmodeler/Repeatmasker $(19,20)$ is a long-standing and comparable annotator of repeats

192 that is more reliant on copy number. With EDTA, $25.8 \%$ of the genome was marked repetitive, with DNA

193 transposons comprised the largest percentage of repeats in the genome, at 16\% (Supplemental Table 3). In

194 contrast, RepeatMasker assessed $36.5 \%$ of the genome as an interspersed repeat, with $28.8 \%$ of the genome being

195 comprised LINE retrotransposons. We merged these repeat annotations with BEDTools (25) to reveal that $38 \%$ of

196 the genome is repetitive. This is in contrast to the repetitive content in red deer, estimated at $22.7 \%$. This

197 difference could be due to technological improvements and could stem from the large proportion of gaps in the 
red deer genome (1.5Gbp) (23). While together these differences could account for a large disparity in

chromosomes (23).

To annotate the genes in the genome we generated 1.5 billion paired end reads of sequencing from six tissues, genome and the gene prediction are of high quality.

\section{Comparison to Related Species}

217 multiple pairs of chromosomes are tandemly fused in B. taurus and vise-versa (Table 2). We confirmed previous reports of chromosome fusions and fissions indicated that twelve cervus chromosomes fused into six in B. taurus, as well as four chromosomes in B. taurus are fused into two cervus chromosomes (Table 2). 
elaphus hippelaphus $\mathrm{Ce} \_\mathrm{Chr} \_8$, a $17 \mathrm{MB}$ region of $\mathrm{Ce} \_\mathrm{Chr} 33$ may have been falsely attached to $\mathrm{Ce}$ _Chr_8 in $\mathrm{C}$. elaphus hippelaphus. Another smaller chromosome translocation of $13.6 \mathrm{MB}$ occurred between Ce_Chr_22 and likely falsely attached to Ce_Chr_3 in C. elaphus hippelaphus. Interestingly, both of these translocations are

227 between chromosomes in elk that are fused chromosomes in B. taurus, Bt_Chr_2 and Bt_Chr_5 (table). While it is 228 possible that these translocations occurred since the divergence of these two species, because the $B$. taurus 229 assembly was used to orient and join scaffolds in the C. elaphus hippelaphus genome assembly, it is likely that 230 these translocations are misassemblies in the C. elaphus hippelaphus genome.

\section{Immune Gene Loss}

232 Nine Bos taurus immune genes were identified from the IMGT GENE-DB database (57) that did not align to the Elk genome: AY644518_TRGJ1, KT723008_IGHJ2, AC172685_IGHA, IMGT000049_TRDC, D16120_TRGJ2, AY149283_IGHJ1, AY2277782_TRAJ31, AY644517_TRG and NW_001494075_IGHJ1. These genes are all components of the T cell receptor: (gamma joining 1), (gamma joining 2), (alpha joining 31), and (delta constant) or of the Immunoglobulin complex: (heavy joining 1), (heavy joining 2) and (heavy constant alpha). Interestingly, seven of the nine genes are located at the very end of chromosomes 01 and 02 in Bos taurus (Table 4). B. taurus

238 chromosomes 01 and 02 have split in C. canadensis into chromosomes 7 and 27, and chromosomes 24 and 15,

239 respectively (Table 2 ) suggesting a possible mechanism for loss of these genes through large chromosomal

240 rearrangements and fission. Future work will be required to investigate how the loss of these genes affects cellular

241 immune response in elk, yet this may provide the foundation necessary to develop long-term immunity to Brucella.

\section{Conclusions}

244 This genome assembly and annotation of the Rocky Mountain elk is the most contiguous assembly of a Cervus

245 species and will serve as an important tool for genomic exploration of all related Cervids. Elk's loss of immune

246 system-related genes in relation to cattle, may provide a clue to establishing a successful vaccination strategy. This 
247 chromosomal assembly of the elk genome will provide an excellent resource for investigating genes involved in

248 elk's poor adaptive cellular immune response to Brucella vaccines.

252 Mary Wood regarding elk sample collection.

254 Conceptualization -SO; data curation - REM, AS; formal analysis - REM, AZ, AS, funding acquisition - SO; investigations - DA, DOB, PB, WE, FT, JWM, JWW; methodology - REM, AZ, AS; resources -- AS, software - REM, AZ, AS; supervision -- SO, validation - REM, AS; visualization -- REM, writing -- REM, AS; review and editing - REM,

Data Availability: The Rocky Mountain elk genome has been deposited at GenBank accession and associated at https://github.com/ISUgenomics/elk_genomics.

1. Stelfox J. Elk in north-west Alberta. Land-Forest-Wildlife. 1964;6(5):14-23.

$2662 . \quad$ Pybus MJ, Butterworth EW, Woods JG. An expanding population of the giant liver fluke 267 (Fascioloides magna) in elk (Cervus canadensis) and other ungulates in Canada. Journal of Wildlife 268 Diseases. 2015;51(2):431-45.

269 3. Green H. The elk of Banff National Park. Unpubl. 1946:32.

270 4. Lloyd H. Transfers f elk for re-stocking. Can Field Nat. 1927;41:126-7.

271 5. Lothian W. A history of Canada's National Parks. 1981;4:155. 
6. Flook DR. A Study of the Apparent Unequal Sex Ration of Wapiti: University of Alberta (Ph. D.); 1967.

7. Stewart KM, Bowyer RT, Kie JG, Cimon NJ, Johnson BK. Temporospatial Distributions of Elk, Mule Deer, and Cattle: Resource Partitioning and Competitive Displacement. Journal of Mammalogy. 2002;83(1):229-44.

8. Cotterill GG, Cross PC, Merkle JA, Rogerson JD, Scurlock BM, Du Toit JT. Parsing the effects of demography, climate and management on recurrent brucellosis outbreaks in elk. Journal of Applied Ecology. 2020;57(2):379-89.

9. Godfroid J. Brucellosis in wildlife. Revue Scientifique et Technique-Office international des épizooties. 2002;21(1):277-86.

10. Lowry J, Goodridge L, Vernati G, Fluegel A, Edwards W, Andrews G. Identification of Brucella abortus genes in elk (Cervus elaphus) using in vivo-induced antigen technology (IVIAT) reveals novel markers of infection. Veterinary microbiology. 2010;142(3-4):367-72.

11. Yingst $S$, Hoover D. T cell immunity to brucellosis. Critical reviews in microbiology. 2003;29(4):313-31.

12. Nol P, Olsen SC, Rhyan JC, Sriranganathan N, McCollum MP, Hennager SG, et al. Vaccination of elk (Cervus canadensis) with Brucella abortus strain RB51 overexpressing superoxide dismutase and glycosyltransferase genes does not induce adequate protection against experimental Brucella abortus challenge. Frontiers in cellular and infection microbiology. 2016;6:10.

13. Putnam NH, O'Connell BL, Stites JC, Rice BJ, Blanchette M, Calef R, et al. Chromosome-scale shotgun assembly using an in vitro method for long-range linkage. Genome research. 2016;26(3):342-50. 14. Lieberman-Aiden E, Van Berkum NL, Williams L, Imakaev M, Ragoczy T, Telling A, et al. Comprehensive mapping of long-range interactions reveals folding principles of the human genome. science. 2009;326(5950):289-93.

15. Zimin AV, Marçais G, Puiu D, Roberts M, Salzberg SL, Yorke JA. The MaSuRCA genome assembler. Bioinformatics. 2013;29(21):2669-77.

16. Dudchenko O, Shamim MS, Batra SS, Durand NC, Musial NT, Mostofa R, et al. The Juicebox Assembly Tools module facilitates de novo assembly of mammalian genomes with chromosome-length scaffolds for under $\$ 1000$. Biorxiv. 2018:254797.

17. Durand NC, Robinson JT, Shamim MS, Machol I, Mesirov JP, Lander ES, et al. Juicebox provides a visualization system for Hi-C contact maps with unlimited zoom. Cell systems. 2016;3(1):99-101.

18. Intitute B. Picard Tools. 2019.

19. Smit A, Hubley R, Green P. RepeatModeler Open-1.0. 2008-2010. Access date Dec. 2014.

20. Smit A, Hubley R, Green P. RepeatMasker Open-4.0. 2013-2015. Institute for Systems Biology http://repeatmasker org. 2015.

21. Li H, Durbin R. Fast and accurate short read alignment with Burrows-Wheeler transform. Bioinformatics. 2009;25(14):1754-60.

22. Dudchenko O, Batra SS, Omer AD, Nyquist SK, Hoeger M, Durand NC, et al. De novo assembly of the Aedes aegypti genome using $\mathrm{Hi}-\mathrm{C}$ yields chromosome-length scaffolds. Science. 2017;356(6333):925.

23. Bana NÁ, Nyiri A, Nagy J, Frank K, Nagy T, Stéger V, et al. The red deer Cervus elaphus genome CerEla1. 0: sequencing, annotating, genes, and chromosomes. Molecular Genetics and Genomics. 2018;293(3):665-84.

24. Madden T. The BLAST sequence analysis tool. The NCBI Handbook [Internet] 2nd edition: National Center for Biotechnology Information (US); 2013.

25. Quinlan AR. BEDTools: the Swiss-army tool for genome feature analysis. Current protocols in bioinformatics. 2014:11.2. 1-.2. 34. 
26. Walker BJ, Abeel T, Shea T, Priest M, Abouelliel A, Sakthikumar S, et al. Pilon: an integrated tool for comprehensive microbial variant detection and genome assembly improvement. PloS one.

321 2014;9(11):e112963.

$322 \quad 27 . \quad$ Biosciences P. SMRT Link. 2017.

323 28. Barnett D, Garrison E, Marth G, Stromberg M. BamTools. 2013.

29. Li H. Minimap and miniasm: fast mapping and de novo assembly for noisy long sequences. Bioinformatics. 2016;32(14):2103-10.

30. Kim D, Langmead B, Salzberg S. HISAT2: graph-based alignment of next-generation sequencing reads to a population of genomes. 2017.

328 31. Li H, Handsaker B, Wysoker A, Fennell T, Ruan J, Homer N, et al. The sequence alignment/map format and SAMtools. Bioinformatics. 2009;25(16):2078-9.

32. Lindenbaum P. JVarkit: java-based utilities for Bioinformatics. 2015. Preprint Available: figshare. 2018.

33. Laetsch DR, Blaxter ML. BlobTools: Interrogation of genome assemblies. F1000Research. 2017;6(1287):1287.

34. Donath A, Jühling F, Al-Arab M, Bernhart SH, Reinhardt F, Stadler PF, et al. Improved annotation of protein-coding genes boundaries in metazoan mitochondrial genomes. Nucleic acids research. 2019;47(20):10543-52.

35. Ou S, Su W, Liao Y, Chougule K, Agda JR, Hellinga AJ, et al. Benchmarking transposable element annotation methods for creation of a streamlined, comprehensive pipeline. Genome biology. 2019;20(1):1-18.

36. Liao Y, Smyth GK, Shi W. The Subread aligner: fast, accurate and scalable read mapping by seedand-vote. Nucleic acids research. 2013;41(10):e108-e. 37. Grabherr MG, Haas BJ, Yassour M, Levin JZ, Thompson DA, Amit I, et al. Full-length transcriptome assembly from RNA-Seq data without a reference genome. Nature biotechnology. 2011;29(7):644-52.

38. Haas BJ, Papanicolaou A, Yassour M, Grabherr M, Blood PD, Bowden J, et al. De novo transcript sequence reconstruction from RNA-seq using the Trinity platform for reference generation and analysis. Nature protocols. 2013;8(8):1494-512.

348 39. Henschel R, Lieber M, Wu L-S, Nista PM, Haas BJ, LeDuc RD, editors. Trinity RNA-Seq assembler performance optimization. Proceedings of the 1st Conference of the Extreme Science and Engineering Discovery Environment: Bridging from the eXtreme to the campus and beyond; 2012.

40. Liu R, Dickerson J. Strawberry: Fast and accurate genome-guided transcript reconstruction and quantification from RNA-Seq. PLOS Computational Biology. 2017;13(11):e1005851. improved reconstruction of a transcriptome from RNA-seq reads. Nature biotechnology. 2015;33(3):290. 42. Pertea M, Kim D, Pertea GM, Leek JT, Salzberg SL. Transcript-level expression analysis of RNAseq experiments with HISAT, StringTie and Ballgown. Nature protocols. 2016;11(9):1650.

43. Song L, Sabunciyan S, Florea L. CLASS2: accurate and efficient splice variant annotation from RNA-seq reads. Nucleic acids research. 2016;44(10):e98-e.

44. Hoff KJ, Lomsadze A, Stanke M, Borodovsky M. BRAKER2: incorporating protein homology information into gene prediction with GeneMark-EP and AUGUSTUS. Plant and Animal Genomes XXVI. 2018.

45. Stanke M, Diekhans M, Baertsch R, Haussler D. Using native and syntenically mapped cDNA alignments to improve de novo gene finding. Bioinformatics. 2008;24(5):637-44.

46. Mapleson D, Venturini L, Kaithakottil G, Swarbreck D. Efficient and accurate detection of splice junctions from RNA-seq with Portcullis. GigaScience. 2018;7(12):giy131. 
47. Venturini L, Caim S, Kaithakottil GG, Mapleson DL, Swarbreck D. Leveraging multiple transcriptome assembly methods for improved gene structure annotation. GigaScience. 2018;7(8):giy093.

48. Consortium U. UniProt: a worldwide hub of protein knowledge. Nucleic acids research. 2019;47(D1):D506-D15.

49. Gremme G. GenomeThreader Gene Prediction Software. 2014.

50. Trapnell C, Roberts A, Goff L, Pertea G, Kim D, Kelley DR, et al. Differential gene and transcript expression analysis of RNA-seq experiments with TopHat and Cufflinks. Nature protocols. 2012;7(3):562. 51. Finn RD, Attwood TK, Babbitt PC, Bateman A, Bork P, Bridge AJ, et al. InterPro in 2017-beyond protein family and domain annotations. Nucleic acids research. 2016;45(D1):D190-D9.

52. Jones $P$, Binns $D$, Chang $H-Y$, Fraser $M$, Li W, McAnulla $C$, et al. InterProScan 5: genome-scale protein function classification. Bioinformatics. 2014;30(9):1236-40.

53. Simão FA, Waterhouse RM, loannidis P, Kriventseva EV, Zdobnov EM. BUSCO: assessing genome assembly and annotation completeness with single-copy orthologs. Bioinformatics. 2015:btv351. 54. Waterhouse RM, Seppey M, Simão FA, Manni M, loannidis P, Klioutchnikov G, et al. BUSCO applications from quality assessments to gene prediction and phylogenomics. Molecular biology and evolution. 2017;35(3):543-8.

55. Rosen BD, Bickhart DM, Schnabel RD, Koren S, Elsik CG, Tseng E, et al. De novo assembly of the cattle reference genome with single-molecule sequencing. GigaScience. 2020;9(3).

56. Wang $Y$, Tang H, DeBarry JD, Tan X, Li J, Wang X, et al. MCScanX: a toolkit for detection and evolutionary analysis of gene synteny and collinearity. Nucleic Acids Research. 2012;40(7):e49-e.

57. Giudicelli V, Chaume D, Lefranc M-P. IMGT/GENE-DB: a comprehensive database for human and mouse immunoglobulin and T cell receptor genes. Nucleic acids research. 2005;33(suppl_1):D256-D61. 58. Zimin AV, Puiu D, Luo M-C, Zhu T, Koren S, Marçais G, et al. Hybrid assembly of the large and highly repetitive genome of Aegilops tauschii, a progenitor of bread wheat, with the MaSuRCA megareads algorithm. Genome research. 2017;27(5):787-92.

59. Proost S, Fostier J, De Witte D, Dhoedt B, Demeester P, Van de Peer Y, et al. i-ADHoRe 3.0-fast and sensitive detection of genomic homology in extremely large data sets. Nucleic acids research. 2011;40(2):e11-e.

\section{Figure 1. Synteny and HiC plot of Elk chromosomes. A. Gene-based synteny between C. elaphus hippelaphus and}

C. canadensis. B. HiC plot of elk chromosomes in JuiceBox. C. Gene-based synteny between B. taurus and C. canadensis. 


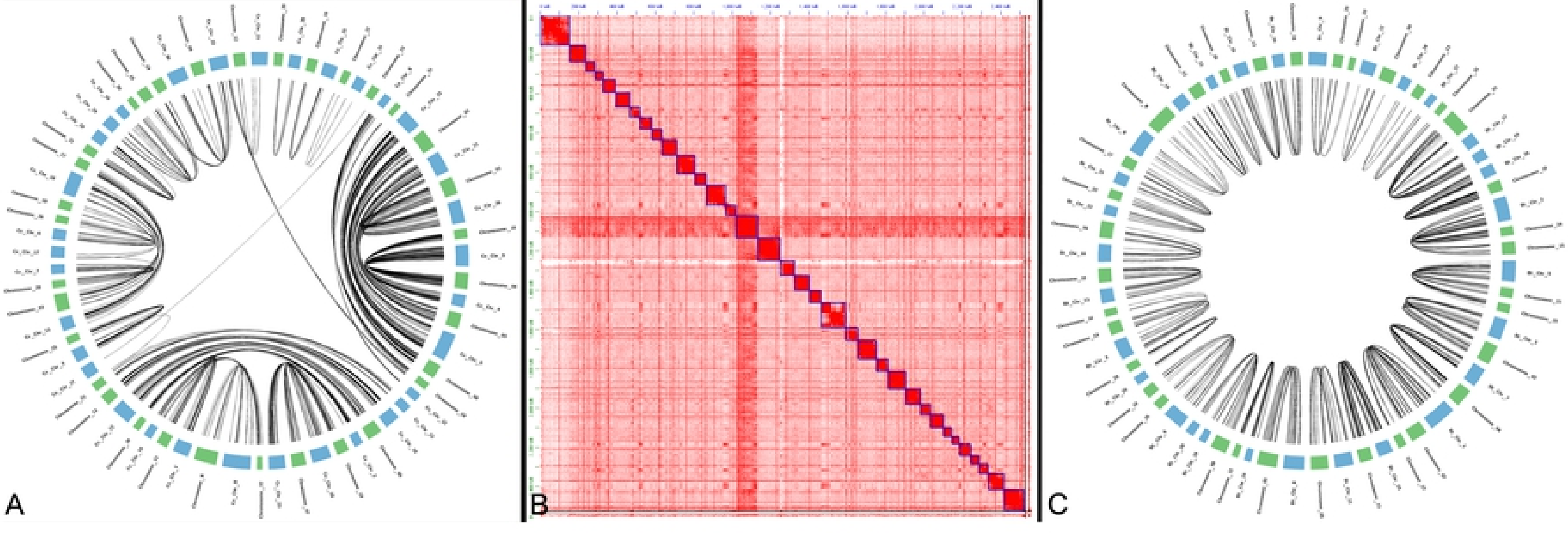

Figure 1 